\title{
Avancées dans la prise en charge de l'adénocarcinome canalaire pancréatique
}

\author{
Grainne M. O'Kane MB BCh BAO MD, Farah Ladak MD MSP, Steven Gallinger MD MSc
}

— Citation : CMAJ 2021 June 7;193:E844-51. doi : 10.1503/cmaj.201450-f

Voir la version anglaise de l'article ici : www.cmaj.ca/lookup/doi/10.1503/cmaj.201450

'incidence de l'adénocarcinome canalaire pancréatique (ACCP) est en hausse et selon les projections, il pourrait devenir la troisième cause de décès par cancer au Canada ${ }^{1}$. Même si on connaît les facteurs de risque d'ACCP, la raison de cette incidence croissante est inconnue. La survie globale à 5 ans pour cette maladie s'est améliorée récemment, mais la survie associée à d'autres cancers a nettement plus augmenté 2 . Selon une étude rétrospective de 2018 sur des patients albertains, plus de $40 \%$ des patients atteints d'un ACCP au stade avancé n'avaient pas été dirigés vers un spécialiste ${ }^{3}$. La résection chirurgicale demeure la seule intervention à visée curative pour l'ACCP, et seulement 15\%-20\% des patients présentent une maladie résécable ${ }^{4}$. Il s'agit toutefois d'une maladie hétérogène et plusieurs critères utiles de classification génomique ${ }^{5,6}$ et sous-types ${ }^{7-9}$ en fonction de l'ARN ont été identifiés et commencent à orienter les stratégies thérapeutiques pour les patients atteints d'une maladie non résécable; et certaines procurent un avantage sur le plan de la survie. Notre point de départ est une synthèse existante du tableau clinique et du traitement chirurgical de l'ACCP10 et nous présentons les données probantes à l'appui des plus récentes avancées du traitement clinique (encadré 1). Une approche pour le traitement de l'ACCP selon chaque stade est présentée à la figure 1 .

\section{Qui est à risque de développer un adénocarcinome canalaire pancréatique?}

Selon des études d'observation, le tabagisme ${ }^{11}$, l'obésité ${ }^{12}$ et un diabète de longue date ${ }^{13}$ sont associés à un risque plus élevé d'ACCP. Des antécédents familiaux de cancer du pancréas chez

Encadré 1 : Données utilisées pour la présente revue Nous avons identifié les articles pour cette revue au moyen d'une interrogation des bases de données MEDLINE, Embase et Cochrane et des références tirées d'articles pertinents, à partir d'une combinaison variée des termes anglais suivants " pancreatic cancer », « pancreas », «PDAC », «PDAC genetics » et «PDAC management and treatment ». Nous avons exclu les articles présentés uniquement sous forme de résumés ou de rapports de réunion, et inclus les articles publiés

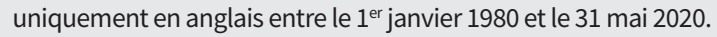

\section{Points clés}

- Les tests de profilage génomique large sont désormais recommandés chez tous les patients atteints d'un adénocarcinome canalaire pancréatique.

- Chez les patients qui y sont candidats, le schéma FOLFIRINOX modifié est la chimiothérapie adjuvante à privilégier, après la résection chirurgicale.

- Les approches néoadjuvantes dans les cas de maladie résécable sont de plus en plus courantes et devraient être envisagées chez les patients qui ont des caractéristiques à haut risque, telles qu'un taux élevé d'antigène glucidique 19.9, au moment du diagnostic.

- Les patients atteints d'un adénocarcinome canalaire pancréatique (ACCP) à la limite de la résécabilité ou localement avancé devraient recevoir une chimiothérapie d'association inductive si possible, avant d'envisager une résection chirurgicale ou un traitement local.

- Si les ressources le permettent, les patients atteints d'un ACCP avancé devraient subir un test de profilage génomique de leur tumeur pour détecter les altérations somatiques que l'on peut cibler à l'aide de traitements.

un parent au premier degré s'observent chez environ $10 \%$ des patients ${ }^{14}$, et chez $5 \%-7 \%$, on retrouve des variants pathogènes de lignée germinale dans les gènes $B R C A 1, B R C A 2$ et $P A L B 2^{15,16}$. Même si le National Comprehensive Cancer Network (NCCN) recommande désormais un dépistage réflexe des lignées germinales à l'aide de tests de profilage génomique large pour les syndromes héréditaires prédisposant au cancer ${ }^{17}$ dès qu'un nouveau diagnostic d'ACCP est posé afin de faciliter la séquence des tests chez les membres de la famille et d'administrer directement les traitements pour la maladie avancée ${ }^{18}$, l'accès à ces tests varie d'une région à l'autre du Canada.

Malgré l'incidence croissante de l'ACCP, le dépistage de la maladie dans la population générale n'est pas recommandé par le récent guide du US Preventive Services Task Force ${ }^{19}$. Chez les patients à risque élevé présentant des syndromes héréditaires prédisposant au cancer, l'échographie endoscopique ou l'imagerie par résonance magnétique sont des modalités de dépistage acceptées ${ }^{20}$; mais, les répercussions du dépistage sur la mortalité dans cette population n’ont pas été établies. 


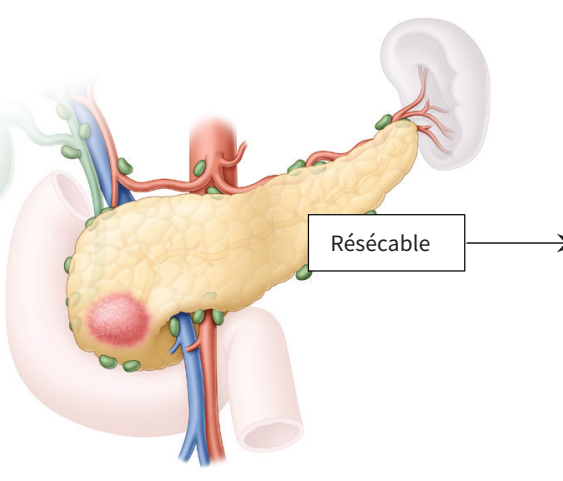
Envise, ganglions régionaux enflés. Envisager une chimiothérapie

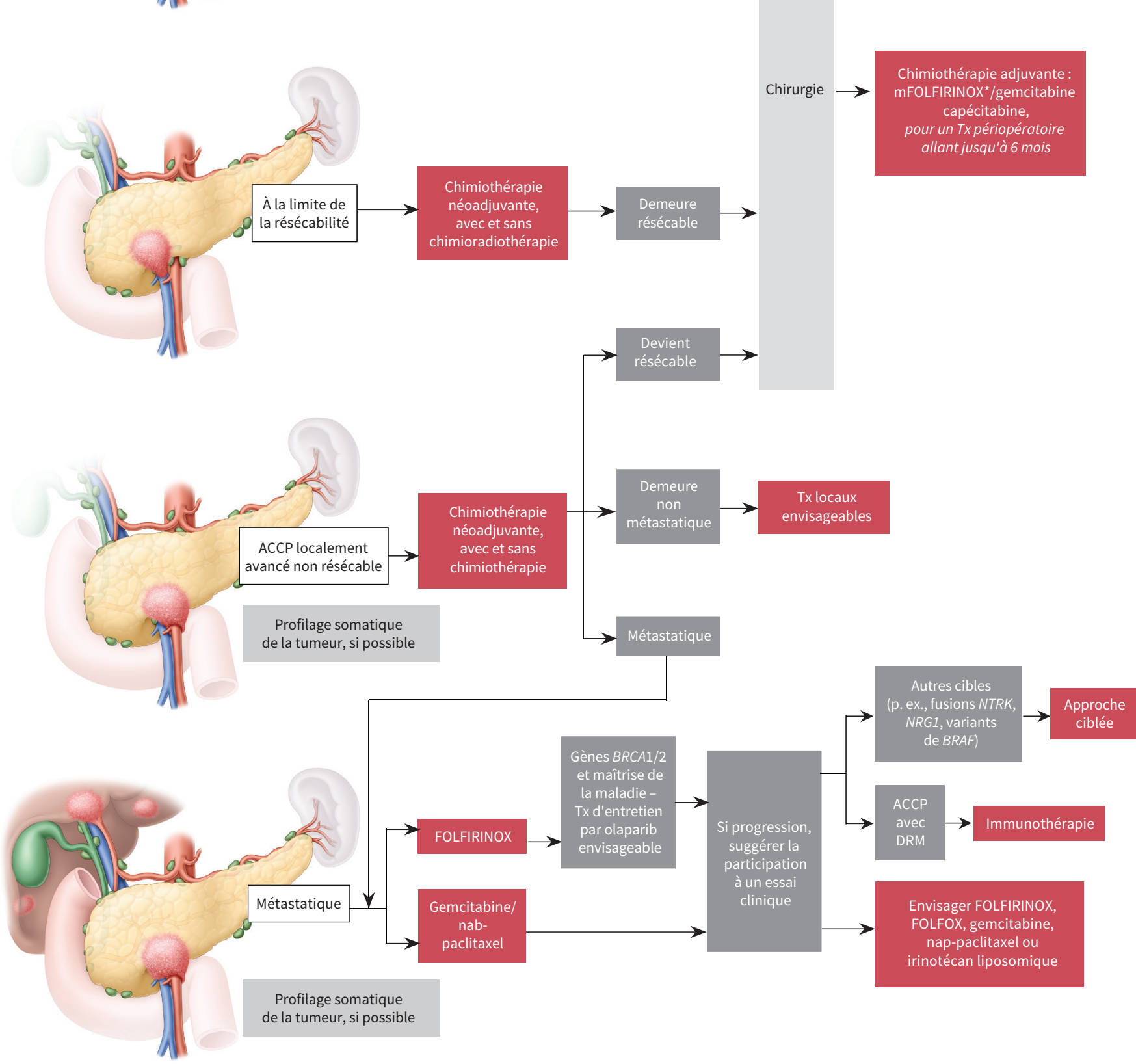

Figure 1 : Approche thérapeutique recommandée pour l'adénocarcinome canalaire pancréatique (ACCP). Si possible, pour tous les cas abordés à l'occasion de la Conférence multidisciplinaire sur le cancer, on préconise un profilage génomique et une participation aux essais cliniques, peu importe le stade de la maladie. Remarque : CA19.9 = antigène glucidique 19.9, DRM = déficit de réparation du mésappariement, FOLFOX = 5-fluorouracile, leucovorine et oxaliplatine, mFOLFIRINOX = FOLFIRINOX modifié (5-fluorouracile, irinotécan, leucovorine et oxaliplatine), Tx = traitement. ${ }^{\star} \mathrm{Chez}$ les patients jugés candidats pour ce schéma thérapeutique. Illustration originale réalisée par l'illustratrice médicale pigiste Christine Kenney. 


\section{Comment l'adénocarcinome canalaire pancréatique est-il diagnostiqué et stadifié?}

La prise en charge de l'ACCP repose sur la détermination de sa résécabilité. La subjectivité de l'interprétation des clichés a mené à la création de modèles types pour les radiologiste ${ }^{21}$. La maladie non métastatique se classe au plan anatomique en 3 groupes: cancer du pancréas résécable, à la limite de la résécabilité et localement avancé non résécable, selon la proximité et l'envahissement du réseau vasculaire veineux ou artériel. Pour établir cette classification, il faut des modalités d'imagerie de grande qualité, préférablement un protocole de tomodensitométrie (TDM) pancréatique multiphasique en coupes minces $^{21}$. Une approche multidisciplinaire et l'expérience cumulée des centres traitant de forts volumes se sont révélées cruciales et ont mené à des changements dans les recommandations thérapeutiques pour près du quart des $\mathrm{cas}^{22}$.

Environ $15 \%-20 \%$ des patients présentent les critères d'un $A C C P$ résécable à l'imagerie ${ }^{4,23}$, défini selon la ligne directrice du NCCN comme une tumeur sans envahissement artériel ou veineux ou ayant un contact de moins de $180^{\circ}$ avec la veine mésentérique supérieure ou la veine porte, sans irrégularités du contour veineux ${ }^{21}$. Dans environ $30 \%$ des nouveaux cas, l'envahissement vasculaire et l'atteinte locale empêcheront toute tentative chirurgicale ${ }^{24}$ et plus de la moitié des patients ont une maladie métastatique pour laquelle la survie médiane, malgré la chimiothérapie, demeure inférieure à 1 an 25,26 .

\section{Quelles sont les options thérapeutiques pour les patients opérables?}

\section{Résection et chimiothérapie adjuvante}

Même si les techniques chirurgicales n'ont pas substantiellement changé depuis une dizaine d'années, pour sa part, le rôle de la chimiothérapie périopératoire évolue (tableau 1). Le traitement adjuvant par gemcitabine a été la norme pendant de nombreuses années ${ }^{29}$ et a permis de doubler la survie à 5 ans comparativement à la chirurgie seule (10,4\% c. 20,7\%). L'étude ESPAC-4 (European Study Group for Pancreatic Cancer-4) a évalué l'utilisation du traitement adjuvant par gemcitabine c. gemcitabine et capécitabine en association et elle a été la première à démontrer la supériorité de la polychimiothérapie, qui a porté la survie globale (SG) médiane à 28 mois $^{28}$. En 2018, les résultats de l'essai clinique à étiquetage en clair de phase III28,33 du Canadian Cancer Trial Group/Unicancer-GI-PRODIGE ont transformé la pratique. Dans cette étude, des patients âgés de moins de 79 ans qui avaient un taux d'antigène glucidique 19.9 (CA19.9) postopératoire de $180 \mathrm{U} / \mathrm{mL}$ ou moins ont obtenu une attribution aléatoire au schéma FOLFIRINOX modifié (5-fluorouracile, irinotécan, leucovorine et oxaliplatine) ou à la gemcitabine seule. La SG médiane a été de 54,5 mois dans le groupe sous FOLFIRINOX modifié c. 35,0 mois dans le groupe sous gemcitabine seule (rapport de risque [HR] 0,64, intervalle de confiance $[I C]$ à $95 \% 0,48-0,86, p=0,003)$, une différence étonnamment marquée comparativement aux autres essais sur l'ACCP. Il faut dire que l'âge médian des patients inscrits à cette étude était de 62 ans, soit nettement moindre que l'âge moyen des patients qui reçoivent un diagnostic d'ACCP (70 ans). Comme prévu, la toxicité a été plus marquée dans le groupe sous FOLFIRINOX modifié. En 2019, l'étude APACT (NabPaclitaxel Plus Gemcitabine Versus Gemcitabine Alone as Adjuvant Therapy in Subjects With Surgically Resected Pancreatic Adenocarcinoma) a évalué l'association gemcitabine-nabpaclitaxel (nanoparticules liées à l'albumine) c. gemcitabine seule et n'a observé aucune amélioration sur le plan de la survie sans maladie ${ }^{27}$.

En général, la chimioradiothérapie adjuvante n'a pas amélioré la survie chez les patients atteints d'ACCP ${ }^{34}$; par contre, on peut l'envisager chez certains patients qui ont des marges de résection positives. Une méta-analyse de 2008 regroupant 4 essais randomisés et contrôlés (ERC) a observé un avantage sur le plan de la survie avec la chimioradiothérapie chez cette cohorte particulière comparativement aux patients qui avaient une résection R0 (c.-à-d., absence de tumeurs microscopiques à $1 \mathrm{~mm}$ de la marge de résection) $)^{35}$.

\section{Chimiothérapie néoadjuvante quand la maladie est résécable}

De tout temps, la chimiothérapie néoadjuvante a été réservée aux patients qui présentaient un cancer du pancréas à la limite de la résécabilité ou localement avancé non résécable. Les médecins acceptent toutefois de plus en plus que l'ACCP est une maladie systémique et on obtient des taux de guérison plus élevés avec la chirurgie et la polychimiothérapie. Malgré cela, une étude de 2014 menée aux États-Unis sur l'effet des complications postopératoires chez les patients sous chimiothérapie adjuvante a noté qu'un peu moins de $50 \%$ des patients soumis à la résection pour un ACCP de stade I-III n'ont pas reçu de chimiothérapie adjuvante ${ }^{36}$. Étant donné l'importance du traitement systémique pour augmenter les chances de guérison, une approche reposant sur la chimiothérapie d'abord pourrait être optimale.

En théorie, la chimiothérapie néoadjuvante permettrait une meilleure sélection biologique parce que $25 \%$ des patients présentent une récurrence dans les 6 mois qui suivent la chirurgie $^{37}$. Ces patients qui ont une récurrence hâtive risquent peu de bénéficier d'une approche chirurgicale. Le traitement néoadjuvant pourrait abaisser le stade de la maladie et accroître la probabilité d'obtenir une résection avec marge négative. Plusieurs établissements aux États-Unis ont adopté cette approche, même si les données des ERC ne sont pas encore disponibles. Le NCCN recommande une approche néoadjuvante uniquement chez les patients dont la maladie est résécable et qui ont des caractéristiques à haut risque, comme un taux élevé de CA19.9, une tumeur volumineuse ou des ganglions régionaux enflés ${ }^{17}$. On trouve au tableau 1 un sommaire des essais sur la chimiothérapie néoadjuvante effectués à ce jour ${ }^{30-32}$. On ne dispose pas encore des conclusions de l'Alliance for Clinical Trials in Oncology (ALLIANCE A021806); mais on y trouvera les différences de résultats entre le schéma FOLFIRINOX modifié néoadjuvant et adjuvant. Un essai canadien de phase II est également en cours (tableau 2). 
Tableau 1 : Essais cliniques de phase II et III sélectionnés sur un traitement adjuvant ou néoadjuvant pour l'adénocarcinome canalaire pancréatique résécable

\begin{tabular}{|c|c|c|c|c|c|c|c|c|c|}
\hline $\begin{array}{l}\text { Groupe } \\
\text { d'auteurs (titre } \\
\text { de l'essai) }\end{array}$ & $\begin{array}{c}\text { Type } \\
\text { d'étude }\end{array}$ & Groupe traité & $\begin{array}{l}\mathrm{N}^{\text {bre de }} \\
\text { patients }\end{array}$ & $\begin{array}{l}\text { Inclusion } \\
\text { spécifique }\end{array}$ & $\begin{array}{l}\text { Paramètre } \\
\text { principal }\end{array}$ & SSM & $\begin{array}{l}\text { Valeur de } \\
\qquad p\end{array}$ & SG & $\begin{array}{l}\text { Valeur } \\
\text { de } p\end{array}$ \\
\hline \multirow{2}{*}{$\begin{array}{l}\text { Conroy et al. }{ }^{25} \\
\text { (PRODIGE24- } \\
\text { CCTG PA6) }\end{array}$} & \multirow[t]{2}{*}{ Phase III } & Gemcitabine & \multirow[t]{2}{*}{493} & \multirow{2}{*}{$\begin{array}{l}\text { ECOG } 0-1 ; \\
\text { Âge, } \leq 76 \text { ans } \\
\text { CA19.9 de } \\
\text { référence } \leq \\
180 \mathrm{U} / \mathrm{mL}\end{array}$} & \multirow[t]{2}{*}{ SSM } & 12,8 & \multirow[t]{2}{*}{$<0,001$} & 35 & \multirow[t]{2}{*}{0,003} \\
\hline & & FOLFIRINOX & & & & 21,6 & & 54,4 & \\
\hline \multirow{2}{*}{$\begin{array}{l}\text { Tempero et al. }{ }^{27} \\
\text { (APACT) }\end{array}$} & \multirow[t]{2}{*}{ Phase III } & Gemcitabine & \multirow[t]{2}{*}{866} & \multirow{2}{*}{$\begin{array}{l}\text { ECOG } 0-1 ; \\
\text { CA19.9< } \\
100 \mathrm{U} / \mathrm{mL}\end{array}$} & \multirow{2}{*}{$\begin{array}{l}\text { SSM par } \\
\text { observateur } \\
\text { indépendant }\end{array}$} & 18,8 & \multirow[t]{2}{*}{0,2} & 36,2 & \multirow[t]{2}{*}{0,045} \\
\hline & & $\begin{array}{l}\text { Gemcitabine plus } \\
\text { nab-paclitaxel }\end{array}$ & & & & 19,4 & & 40,5 & \\
\hline \multirow{2}{*}{$\begin{array}{l}\text { Neoptomelos et } \\
\text { al. }{ }^{28} \text { (ESPAC-4) }\end{array}$} & \multirow[t]{2}{*}{ Phase III } & Gemcitabine & \multirow[t]{2}{*}{730} & \multirow[t]{2}{*}{$\mathrm{SF}-\mathrm{OMS}<2$} & \multirow[t]{2}{*}{ SG } & 13,1 & \multirow[t]{2}{*}{0,08} & 25,5 & \multirow[t]{2}{*}{0,032} \\
\hline & & $\begin{array}{l}\text { Gemcitabine plus } \\
\text { capécitabine }\end{array}$ & & & & 13,9 & & 28,8 & \\
\hline \multirow{2}{*}{$\begin{array}{l}\text { Oettle et al. }{ }^{29} \\
\text { (CONKO-001) }\end{array}$} & \multirow[t]{2}{*}{ Phase III } & Observation & \multirow[t]{2}{*}{354} & \multirow{2}{*}{$\begin{array}{l}\text { Karnofsky SF } \\
\geq 50 \%\end{array}$} & \multirow[t]{2}{*}{ SSM } & 6,7 & \multirow[t]{2}{*}{$<0,001$} & 20,2 & \multirow[t]{2}{*}{0,01} \\
\hline & & Gemcitabine & & & & 13,4 & & 22,8 & \\
\hline \multicolumn{10}{|c|}{ Essais sur des néoadjuvants } \\
\hline $\begin{array}{l}\text { Motoi et al. }{ }^{30} \\
\text { (Prep-02/JSAP-05) }\end{array}$ & \multirow[t]{2}{*}{$\begin{array}{l}\text { Phase II/ } \\
\text { III }\end{array}$} & $\begin{array}{l}\text { Gemcitabine/ } \\
\text { S1-chirurgie-S1 }\end{array}$ & 364 & \multirow{2}{*}{$\begin{array}{l}\text { Résécable et } \\
\text { LR; } \\
\text { ECOG 0-1 }\end{array}$} & \multirow[t]{2}{*}{ SG } & NR & - & 36,7 & \multirow[t]{2}{*}{0,015} \\
\hline Résumé & & Chirurgie-S1 & & & & & & 26,6 & \\
\hline \multirow[t]{2}{*}{$\begin{array}{l}\text { Versteijne et al. }{ }^{31} \\
\text { (PREOPANC) }\end{array}$} & \multirow[t]{2}{*}{ Phase III } & $\begin{array}{l}\text { Gemcitabine/ } \\
\text { radiothérapie- } \\
\text { chirurgie- } \\
\text { gemcitabine }\end{array}$ & 246 & \multirow[t]{2}{*}{$\begin{array}{l}\text { Résécable et } \\
\text { LR }\end{array}$} & $\begin{array}{l}\text { SG selon } \\
\text { l'intention de } \\
\text { traiter }\end{array}$ & 8,1 & 0,03 & 16 & 0,096 \\
\hline & & $\begin{array}{l}\text { Chirurgie- } \\
\text { gemcitabine }\end{array}$ & & & & 7,7 & & 14,3 & \\
\hline $\begin{array}{l}\text { Sohal et al. }{ }^{32} \\
\text { (SWOG 1505) } \\
\text { Résumé }\end{array}$ & Phase II & $\begin{array}{l}\text { FOLFIRINOX } \\
\text { modifié-chirurgie- } \\
\text { FOLFIRINOX } \\
\text { modifié }\end{array}$ & 102 & Résécable & SG à 2 ans & $10,9^{\star}$ & NR & 22,4 & NS \\
\hline & & $\begin{array}{l}\text { Gemcitabine/ } \\
\text { nab-paclitaxel- } \\
\text { chirurgie- } \\
\text { gemcitabine/ } \\
\text { nab-paclitaxel }\end{array}$ & & & & $14,2^{\star}$ & & 23,6 & \\
\hline
\end{tabular}

Remarque : CA19.9 = antigène glucidique 19.9, ECOG = Eastern Cooperative Oncology Group, LR = limite de la résécabilité, NR = non rapporté, NS = non significatif, OMS = Organisation mondiale de la santé, $\mathrm{S} 1$ = type de chimiothérapie, SF = statut fonctionnel, SG = survie globale, SSM = survie sans maladie.

*La SSM commence au moment de la chirurgie.

\section{Radiothérapie périopératoire}

Le rôle de la radiothérapie préopératoire demeure incertain dans les cas d'ACCP résécable. L'essai PREOPANC (Preoperative chemoradiotherapy versus immediate surgery for resectable and borderline resectable pancreatic cancer) ${ }^{31}$ a fait état des résultats de la chimioradiothérapie néoadjuvante (gemcitabine) comparativement à la chirurgie d'abord, suivie de gemcitabine en traitement adjuvant chez des patients présentant une maladie résécable ou à la limite de la résécabilité. Plus de la moitié des patients inscrits avaient un ACCP résécable. La survie globale ne s'est pas améliorée. Par contre, la chimioradiothérapie préopératoire a été associée à une meilleure survie sans maladie. En outre, chez les patients soumis à la chirurgie et à la chimiothérapie adjuvante, la survie a été meilleure que dans le groupe sous traitement néoadjuvant (35 mois c. 20 mois).

\section{Maladie à la limite de la résécabilité}

Les patients sont considérés comme atteints d'un ACCP à la limite de la résécabilité si la tumeur touche des artères majeures (p. ex., tronc cœliaque, artère mésentérique supérieure ou artère hépatique commune), mais à moins de $180^{\circ}$ de circonférence. La veine porte ou la veine mésentérique supérieure peuvent être touchées dans l'ACCP à la limite de la résécabilité, dans la mesure où le contour du vaisseau est préservé17. Longtemps, l'atteinte du réseau vasculaire a été considérée comme un critère d'exclusion pour la chirurgie à visée curative puisqu'il était difficile d'obtenir des marges négatives ${ }^{39}$ et que le risque de propagation micrométastatique était plus élevé ${ }^{40}$. Or, plusieurs études qui ont évalué les approches néoadjuvantes ont depuis remis en cause cette convention en montrant une conversion vers la résécabilité et des résultats semblables 
Tableau 2 : Essais de phase II et III en cours ou complétés sur l'adénocarcinome canalaire pancréatique sélectionnés pour des questions spécifiques

\begin{tabular}{|c|c|c|c|c|c|c|}
\hline $\begin{array}{l}\text { Titre ( } n^{\circ} \text { d'essai } \\
\text { clinique) }\end{array}$ & $\begin{array}{c}\text { Type } \\
\text { d'étude }\end{array}$ & Groupe traité & $\begin{array}{c}\mathbf{N}^{\text {bre de }} \\
\text { patients } \\
\text { prévu }\end{array}$ & $\begin{array}{l}\text { Inclusion } \\
\text { spécifique }\end{array}$ & Question & $\begin{array}{c}\text { Paramètre } \\
\text { principal }\end{array}$ \\
\hline
\end{tabular}

\section{ACCP résécable ou à la limite de la résécabilité}

\begin{tabular}{lll} 
ALLIANCE (A02180 PAC3) & Phase III & $\begin{array}{l}\text { FOLFIRINOX modifié en } \\
\text { période périopératoire c. } \\
\text { chirurgie et FOLFIRINOX } \\
\text { modifié en Tx adjuvant }\end{array}$ \\
\hline PANDAS-PRODIGE-44 & Phase II $\quad \begin{array}{l}\text { FOLFIRINOX-chirurgie- } \\
\text { (NCT02676349) }\end{array}$ & $\begin{array}{l}\text { FOLFIRINOX en } \\
\text { chimiothérapie adjuvante- } \\
\text { chimioradiothérapie- } \\
\text { (capécitabine/50.4Gy)- } \\
\text { chirurgie-chimiothérapie } \\
\text { adjuvante }\end{array}$
\end{tabular}

$344 \quad \begin{array}{ll}\text { ACCP } \\ \text { résécable }\end{array} \quad \begin{aligned} & \text { L'approche néoadjuvante SG } \\ & \text { est-elle supérieure à la } \\ & \text { chirurgie suivie d'un Tx } \\ & \text { adjuvant? }\end{aligned}$

\section{ACCP localement avancé}

$\begin{array}{lll}\begin{array}{l}\text { CROSSFIRE } \\ \text { (NCT02791503) }\end{array} & \text { Phase II } & \begin{array}{l}\text { FOLFIRINOX et Él; } \\ \text { FOLFIRINOX et RSA }\end{array} \\ \begin{array}{l}\text { CONKO-007 } \\ \text { (NCT01827553) }\end{array} & \text { Phase III } & \begin{array}{l}\text { Chimiothérapie d'induction } \\ \text { avec gemcitabine ou } \\ \text { FOLFIRINOX, suivie de } \\ \text { radiothérapie; chimiothérapie } \\ \text { d'induction avec gemcitabine } \\ \text { ou FOLFIRINOX seul }\end{array}\end{array}$

\section{ACCP avancé ou métastatique}

CCTG PA.738 Phase I

PANC003 (NCT03504423) Phase III

PASS-01 (NCT04469556)
Gemcitabine/nab-paclitaxel; Gemcitabine/nab-paclitaxel plus durvalumab plus trémélimumab

FOLFIRINOX modifié; FOLFIRINOX modifié et CPI-613

FOLFIRINOX modifié; Gemcitabine/nab-paclitaxel
Résécable et

LR;

ECOG 0 ou 1;

Âge,

$18-75$ ans
La chimioradiothérapie additionnelle conforme en période préopératoire améliore-t-elle les taux de résection $\mathrm{R} 0$ ?

Après FFX, quelle modalité est la plus efficace, l'Él ou la RSA?

$\begin{array}{ll}830 \quad \mathrm{CPLA} ; & \text { La chimioradiothérapie } \\ & \text { en post-chimiothérapie } \\ & \text { d'induction se compare- } \\ & \text { t-elle à la chimiothérapie } \\ & \text { seule pour améliorer les } \\ & \text { résultats du CPLA? }\end{array}$
ECOG $0 / 1$
Maladie
mesurable 500 Stade IV; ECOG 0/1
Stade IV; absence de mutation des cellules germinales
L'ajout du PD-L1 en association et l'inhibition de la CTLA-4 ajoutée à la chimiothérapie améliorent-ils la survie?

Le CPI 613, qui cible le cycle tricarboxylique mitochondrial, améliore-t-il les résultats dans le cancer pancréatique métastatique?

FOLFIRINOX modifié est-il supérieur à la gemcitabine/nabpaclitaxel dans le traitement du cancer du pancréas de stade IV?
Taux de résection $\mathrm{RO}$ 
de 37,7 mois, ce qui suggère un avantage associé à cette approche ${ }^{49}$. Le rôle de la radiothérapie stéréotaxique corporelle dans l'ACCP à la limite de la résécabilité reste à vérifier. L'essai ALLIANCE A021501 en cours souhaite comparer le traitement par FOLFIRINOX avec et sans radiothérapie stéréotaxique corporelle pour la prise en charge préopératoire des tumeurs à la limite de la résécabilité (ClinicalTrials.gov, n NCT02839343); par contre, le groupe soumis à la radiothérapie stéréotaxique corporelle dans le cadre de cette étude a récemment été aboli en raison de sa futilité, et les résultats finaux sont attendus.

\section{Comment devrait-on soigner les patients atteints d'une maladie localement avancée?}

Environ $30 \%$ des patients atteints d'un ACCP ont une maladie localement avancée non résécable, avec envahissement vasculaire substantiel au moment du diagnostic ${ }^{24}$, ce qui est généralement une contre-indication à la chirurgie à visée curative. L'essai SCALOP (Selective Chemoradiation in Advanced LOcalised Pancreatic Cancer) ${ }^{50}$ et une revue systématique qualitative de $2009^{51}$ ont fait état de métastases subcliniques chez $50 \%$ des patients que l'on croyait à l'origine atteints seulement d'une maladie localement avancée à partir des épreuves d'imagerie transversale. La chimiothérapie d'induction peut, par conséquent, donner du temps, permettre l'identification des patients susceptibles de progresser et la sélection des traitements appropriés ${ }^{50,51}$.

\section{Traitements systémiques}

Aucune recommandation standard n'existe pour le traitement systémique initial chez les patients atteints d'un cancer du pancréas localement avancé non résécable. La plupart des établissements ont surtout utilisé les schémas pour la maladie métastatique chez les patients atteints d'une maladie localement avancée non résécable en raison de l'absence de solides données probantes pour cette catégorie de patients. Une revue systématique et méta-analyse de 2016 sur FOLFIRINOX regroupant 689 patients atteints d'un cancer du pancréas localement avancé non résécable a fait état d'une SG médiane de 24,2 mois ${ }^{52}$. Plusieurs de ces patients ont reçu un traitement local additionnel après la chimiothérapie, et le quart ont en fait bien répondu à la chimiothérapie et ont pu subir une résection chirurgicale. L'étude LAPACT, une étude internationale de phase II à étiquetage en clair multicentrique sur le nab-paclitaxel (Abraxane) plus gemcitabine chez des patients atteints d'un cancer du pancréas localement avancé (CPLA) a évalué ce schéma et constaté une réponse de 33,6\%. Au cours de cette étude, $16 \%$ des patients ont subi une chirurgie, et 7 ont bénéficié d'une résection avec des marges microscopiques négatives ${ }^{53}$. L'ajout d'agents novateurs pour abaisser le stade de la maladie et permettre la chirurgie est un domaine de recherche actif (tableau 2).

\section{Traitements locaux}

Les patients atteints d'un ACCP localement avancé non résécable peuvent présenter une morbidité considérable. La douleur abdominale rebelle et la sténose du défilé gastrique sont les 2 plus fréquentes causes d'hospitalisation ${ }^{54}$. Jusqu'à $33 \%$ des patients atteints d'un cancer du pancréas localement avancé non résécable décéderont des complications liées à la progression locale, telle qu'hémorragie et perforation ${ }^{55}$. La chimioradiothérapie ou la radiothérapie stéréotaxique corporelle administrée après la chimiothérapie dans le but de consolider les réponses, n'ont pas encore montré qu'elles amélioraient la SG. On peut difficilement diriger les doses de radiothérapie vers les tumeurs pancréatiques en raison de leur proximité avec les organes creux telles que l'intestin grêle et l'estomac; la radiothérapie dirigée vers ces organes peut entraîner une toxicité aiguë ${ }^{56}$. Le groupe Stanford recrute actuellement des patients pour une étude de phase III sur le schéma FOLFIRINOX modifié, avec et sans radiothérapie stéréotaxique corporelle, pour le cancer du pancréas localement avancé non résécable (tableau 2).

Parmi les autres traitements locaux en cours d'évaluation pour la maladie localement avancée non résécable, mentionnons l'électroporation irréversible, une technique non thermique de destruction locale qui génère un courant direct à travers la tumeur ${ }^{57}$. Des études rétrospectives sur l'innocuité et l'efficacité de l'électroporation irréversible pour le cancer du pancréas localement avancé non résécable ont donné des résultats mitigés, et une revue systématique de 2019 a fait état de morbidité associée au traitement chez 1 patient sur $3^{58}$. L'étude multicentrique prospective PANFIRE-2 (Percutaneous Irreversible Electroporation in Locally Advanced and Recurrent Pancreatic Cancer), au cours de laquelle des patients atteints d'un cancer du pancréas localement avancé non résécable recevaient soit une chimiothérapie d'induction à base de gemcitabine soit FOLFIRINOX suivi de l'électroporation irréversible ${ }^{59}$, a fait état d'une SG de 17 mois à partir du diagnostic dans les 2 groupes. Des essais randomisés et contrôlés doivent être effectués si on veut déterminer le rôle de l'électroporation irréversible; une étude de faisabilité recrute actuellement des patients au Réseau universitaire de santé de Toronto (ClinicalTrials.gov, n NCT03257150).

\section{Quels progrès ont été réalisés dans le traitement de la maladie métastatique?}

Les patients atteints d'un ACCP devraient être encouragés à participer aux essais cliniques sur des traitements. Les schémas standard de première intention incluent FOLFIRINOX ${ }^{25}$ modifié et gemcitabine-nab-paclitaxel ${ }^{26}$. Le traitement par gemcitabinenab-paclitaxel n'est remboursé qu'à titre d'agent de première intention dans la plupart des provinces canadiennes et ne l'est pas si d'autres agents ont été préalablement essayés, ce qui limite les options dans la séquence des traitements envisageables. Le seul schéma de seconde intention à avoir montré une amélioration de la survie chez les patients est l'association irinotécan liposomique/5-fluorouracile (essai NAPOLI-1), qui n'est pas remboursée actuellement par les régimes publics au Canada ${ }^{60}$. Même si aucun ERC n'a encore été fait pour comparer les schémas FOLFIRINOX modifié et gemcitabine-nab-paclitaxel, l'essai nord-américain multicentrique PASS-01 (Pancreatic Adenocarcinoma Signature Stratification for Treatment-01) recrute actuellement (ClinicalTrials.gov, nº NCT04469556). Cette étude vise à établir des biomarqueurs prédictifs pour ces 2 schémas 
(tableau 2). Une récente étude a montré qu'un sous-type d'ACCP à ARN basaloïde est plus résistant à la chimiothérapie et, en particulier, à FOLFIRINOX ${ }^{61}$.

La présence d'un variant pathogène de lignée germinale dans les gènes $B R C A 1, B R C A 2$ ou PALB2 est actuellement le seul biomarqueur moléculaire qui peut orienter les médecins vers des schémas à base de platine, comme FOLFIRINOX modifié. Chez les patients porteurs de ces biomarqueurs, la tumeur risque d'être inapte pour la réparation de la recombinaison homologue, c'està-dire incapable de réparer les cassures de l'ADN double brin. Des schémas à base de platine comme FOLFIRINOX ou cisplatine et gemcitabine devraient être envisagés ${ }^{18}$ parce que le platine exploite cette faiblesse de la tumeur. L'essai POLO (Pancreas Cancer Olaparib Ongoing) de 2019 a mené à l'approbation par Santé Canada de l'olaparib, un inhibiteur de la poly(ADP-ribose) polymérase, comme approche au traitement d'entretien chez les patients porteurs d'un variant pathogène de lignée germinale dans les gènes $B R C A 1$ ou $B R C A 2$, qui a donné lieu à une maîtrise de la maladie après 4 mois de traitement à base de platine. Dans cet essai international de phase III, 154 patients ont obtenu une attribution aléatoire à un traitement d'entretien par olaparib c. placebo (3:2), et les patients sous traitement actif ont présenté une meilleure survie sans maladie, mais aucune différence sur le plan de la SG ni amélioration de la qualité de vie ${ }^{18}$. Même si l'olaparib a été approuvé par Santé Canada, son utilisation n'est pas remboursée par les régimes publics à l'heure actuelle et les patients doivent en assumer les frais.

\section{Quels sont les traitements ciblés émergents pour l'ACCP?}

Quatre-vingt-dix pour cent des ACCP hébergent une mutation $K R A S$ oncogène pour laquelle aucun agent ciblé n'avait encore été mis au point avec succès. Mais un variant spécifique de l'oncogène KRAS au niveau du codon 12 (G12C), qui s'observe dans $1 \%-2 \%$ des cas d'ACCP, se révèle prometteur comme cible potentielle. En 2019, l'essai CodeBreak 100 a fait état d'une maîtrise de la maladie chez 9 patients sur 11 ayant reçu un agent ciblant le $\mathrm{G}_{12} \mathrm{C}^{64}$. Cette étude ouvre la porte à plus d'inhibiteurs de l'oncogène KRAS, ce qui pourrait transformer les traitements. En outre, les changements du nombre de copies d'ADN dans le gène KRAS mutant pourraient modifier l'agressivité de certaines tumeurs et l'hypoxie tumorale fréquente pourrait atténuer les réponses au traitement dans l'ACCP ${ }^{62,63}$. Plusieurs autres cibles somatiques peu fréquentes que l'on pourrait éventuellement viser figurent parmi les ACCP à gène KRAS de type sauvage, qui représentent environ $5 \%-10 \%$ des $\operatorname{cas}^{65,66}$. Les fusions de gènes impliquant $N T R K^{67}, N R G 1^{68,69}, R E T^{69}$ et $A L K^{70}$ sont importantes pour identifier ce sous-type d'ACCP. De plus, des altérations du gène $B R A F$ ont été signalées dans l'ACCP à gène KRAS de type sauvage ${ }^{65,66,71}$. En 2020, l'essai portant sur le registre Know Your Tumor a fait état d'un avantage sur la survie lorsque les patients et les traitements étaient assortis, ce qui souligne l'importance du profilage somatique des tumeurs ${ }^{72}$.

Le stroma desmoplastique caractéristique qui enveloppe les cellules tumorales pancréatiques peut aussi promouvoir la tumorigenèse et inhiber la réponse au traitement systémique. Plusieurs agents ciblant le stroma ont échoué lors d'essais cliniques, même si d'autres sont en cours d'investigation ${ }^{73}$. Le volumineux essai de phase III HALO 109-301, sur l'ajout de PEGPH20 (un agent biologique) au schéma gemcitabine-nab-paclitaxel, n'a démontré aucun avantage pour ce qui est des tumeurs ayant une forte expression d'acide hyaluronique, malgré des données de phase II prometteuses ${ }^{74}$.

L'adénocarcinome canalaire pancréatique est considéré comme une tumeur « froide » au plan immunologique, car peu de lymphocytes infiltrent la tumeur et le fardeau tumoral mutationnel est faible.

Selon une hypothèse, l'association d'une chimiothérapie avec des inhibiteurs du point de contrôle immunitaire pourrait stimuler le système immunitaire et induire une antigénicité. Toutefois, l'essai randomisé PA.7, du Canadian Cancer Trials Group (CCTG) a révélé en 2020 qu'il n'y avait aucun avantage à ajouter le trémélimumab et le durvalumab au schéma gemcitabine-nabpaclitaxel en traitement de première intention chez les patients porteurs d'une maladie métastatique ${ }^{38}$. La SG médiane a été de 8,8 mois, contre 9,8 mois pour le groupe expérimental $(p=0,7)$.

D'autres stratégies visant à susciter une réponse immunitaire incluent les vaccins et l'immunothérapie adoptive; toutefois, ces modalités ne se sont pas révélées efficaces dans les cas d'ACCP. À noter, les ACCP qui présentent une anomalie de la réparation des mésappariements de l'ADN sont des tumeurs " chaudes", très immunogènes. Elles représentent moins de $1 \%$ de ces tumeurs; les tumeurs chaudes peuvent émaner de variants pathogènes de lignée germinale défectueuse dans les gènes de réparation des mésappariements, aussi connus sous le nom de syndrome de Lynch, ou en raison d'altérations somatiques de ces gènes ${ }^{75}$. Le déficit de réparation du mésappariement de I'ADN est devenu le premier biomarqueur agnostique pour lequel a été approuvé le pembrolizumab (un inhibiteur des points de contrôle immunitaires du récepteur PD-1 [Programmed Cell Death Protein- 1$)^{76}$. Sur les 86 patients d'une étude déterminante, 8 avaient déjà été traités pour un ACCP avancé et 5 ont répondu au pembrolizumab, dont 2 ont manifesté des réponses complètes $^{76}$. Toutefois, l'essai KEYNOTE-158 de 2020, qui incluait 22 patients présentant un ACCP avec déficit de réparation du mésappariement de l'ADN, a fait état de seulement 4 patients ayant présenté une réponse documentée ( 1 réponse complète et 3 réponses partielles, taux de réponse objective 18,2\%) ${ }^{77}$. Malgré un robuste biomarqueur du déficit de réparation du mésappariement de l'ADN, les patients canadiens ont un accès limité aux inhibiteurs du récepteur PD-1 et doivent souvent se tourner vers les essais cliniques pour y avoir accès.

\section{Conclusion}

L'amélioration des résultats a été lente chez les patients atteints d'ACCP, même si d'importantes percées scientifiques ont permis au corps médical de mieux comprendre cette maladie mortelle. Les patients canadiens ont un accès limité aux nouveaux médicaments qui pourraient améliorer les résultats et on connaît trop peu les options thérapeutiques. Le rôle de la chimiothérapie néoadjuvante 
dans l'ACCP résécable a été défini. Le profilage des lignées germinales et somatiques permettra d'identifier d'autres patients susceptibles de bénéficier d'une approche personnalisée pour les tumeurs sensibles à des traitements susceptibles de prolonger la survie. Il faudra approfondir la recherche en cours sur l'interconnexion complexe entre tumeur, stroma et microenvironnement tumoral pour mieux sélectionner les agents qui ciblent ces compartiments. Les médecins canadiens devraient diriger les patients atteints d'ACCP vers des spécialistes, encourager leur participation à des essais cliniques et soutenir la recherche collaborative sur de nouvelles modalités thérapeutiques.

\section{Références}

1. Brenner DR, Weir HK, Demers AA, et al. Projected estimates of cancer in Canada in 2020. CMAJ 2020;192:E199-205.

2. Cancer facts and figures 2020. Atlanta: American Cancer Society; 2020. Accessible ici : www.cancer.org/content/dam/cancer-org/research/cancer-facts-and -statistics/annual-cancer-facts-and-figures/2020/cancer-facts-and-figures-2020 .pdf (consulté le 9 oct. 2020).

3. Abdel-Rahman O, Xu Y, Tang PA, et al. A real-world, population-based study of patterns of referral, treatment, and outcomes for advanced pancreatic cancer. Cancer Med 2018;7:6385-92.

4. Vincent A, Herman J, Schulick R, et al. Pancreatic cancer. Lancet 2011;378:607-20.

5. Waddell N, Pajic M, Patch AM, et al. Whole genomes redefine the mutational landscape of pancreatic cancer. Nature 2015;518:495-501.

6. Connor AA, Denroche RE, Jang GH, et al. Association of distinct mutational signatures with correlates of increased immune activity in pancreatic ductal adenocarcinoma. JAMA Oncol 2017;3:774-83.

7. Moffitt RA, Marayati R, Flate EL, et al. Virtual microdissection identifies distinct tumor- and stroma-specific subtypes of pancreatic ductal adenocarcinoma. Nat Genet 2015;47:1168-78.

8. Collisson EA, Sadanandam A, Olson P, et al. Subtypes of pancreatic ductal adenocarcinoma and their differing responses to therapy. Nat Med 2011;17:500-3.

9. Bailey P, Chang DK, Nones K, et al. Genomic analyses identify molecular subtypes of pancreatic cancer. Nature 2016;531:47-52.

10. Kanji ZS, Gallinger S. Diagnosis and management of pancreatic cancer. CMAJ 2013;185:1219-26.

11. Bosetti C, Lucenteforte E, Silverman DT, et al. Cigarette smoking and pancreatic cancer: an analysis from the International Pancreatic Cancer Case-Control Consortium (Panc4). Ann Oncol 2012;23:1880-8.

12. Aune D, Greenwood DC, Chan DS, et al. Body mass index, abdominal fatness and pancreatic cancer risk: a systematic review and non-linear dose-response meta-analysis of prospective studies. Ann Oncol 2012;23:843-52.

13. Bosetti C, Rosato V, Li D, et al. Diabetes, antidiabetic medications, and pancreatic cancer risk: an analysis from the International Pancreatic Cancer CaseControl Consortium. Ann Oncol 2014;25:2065-72.

14. Klein AP, Brune KA, Petersen GM, et al. Prospective risk of pancreatic cancer in familial pancreatic cancer kindreds. Cancer Res 2004;64:2634-8.

15. Holter S, Borgida A, Dodd A, et al. Germline BRCA mutations in a large clinic-based cohort of patients with pancreatic adenocarcinoma. J Clin Oncol 2015;33:3124-9.

16. Golan T, Kindler HL, Park JO, et al. Geographic and ethnic heterogeneity of germline BRCA1 or BRCA2 mutation prevalence among patients with metastatic pancreatic cancer screened for entry into the POLO trial. J Clin Oncol 2020;38:1442-54.

17. Network NCC: NCCN Guidelines with NCCN Evidence Blocks. Plymouth Meeting (PA): National Comprehensive Cancer Network. Accessible ici : https://www. nccn.org/professionals/physician_gls/pdf/pancreatic_blocks.pdf (consulté le 9 sept. 2020). Connexion requise.

18. Golan T, Hammel P, Reni M, et al. Maintenance olaparib for germline BRCAmutated metastatic pancreatic cancer. N Engl J Med 2019;381:317-27.

19. US Preventive Services Task Force; Owens DK, Davidson KW, Krist AH, et al. Screening for pancreatic cancer: US Preventive Services Task Force reaffirmation recommendation statement. JAMA 2019;322:438-44.

20. Aslanian HR, Lee JH, Canto MI. AGA clinical practice update on pancreas cancer screening in high-risk individuals: expert review. Gastroenterology 2020;159:358-62.
21. Al-Hawary MM, Francis IR, Chari ST, et al. Pancreatic ductal adenocarcinoma radiology reporting template: consensus statement of the Society of Abdominal Radiology and the American Pancreatic Association. Radiology 2014;270:248-60.

22. Pawlik TM, Laheru D, Hruban RH, et al. Evaluating the impact of a single-day multidisciplinary clinic on the management of pancreatic cancer. Ann Surg Oncol 2008;15:2081-8.

23. Li D, Xie K, Wolff R, et al. Pancreatic cancer. Lancet 2004;363:1049-57.

24. Gurusamy KS, Kumar S, Davidson BR, et al. Resection versus other treatments for locally advanced pancreatic cancer. Cochrane Database Syst Rev 2014; CD010244.

25. Conroy T, Desseigne F, Ychou M, et al. FOLFIRINOX versus gemcitabine for metastatic pancreatic cancer. N Engl J Med 2011;364:1817-25.

26. Von Hoff DD, Ervin T, Arena FP, et al. Increased survival in pancreatic cancer with nab-paclitaxel plus gemcitabine. N Engl J Med 2013;369:1691-703.

27. Tempero MA, Reni M, Riess H, et al. APACT: phase III, multicenter, international, open-label, randomized trial of adjuvant nab-paclitaxel plus gemcitabine (nab-P/G) vs gemcitabine $(G)$ for surgically resected pancreatic adenocarcinoma. J Clin Oncol 2019;37(Suppl 15):4000.

28. Neoptolemos JP, Palmer DH, Ghaneh P, et al. Comparison of adjuvant gemcitabine and capecitabine with gemcitabine monotherapy in patients with resected pancreatic cancer (ESPAC-4): a multicentre, open-label, randomised, phase 3 trial. Lancet 2017;389:1011-24.

29. Oettle H, Post $\mathrm{S}$, Neuhaus $\mathrm{P}$, et al. Adjuvant chemotherapy with gemcitabine vs observation in patients undergoing curative-intent resection of pancreatic cancer: a randomized controlled trial. JAMA 2007;297:267-77.

30. Motoi F, Kosuge T, Ueno $\mathrm{H}$, et al. Randomized phase II/III trial of neoadjuvant chemotherapy with gemcitabine and S-1 versus upfront surgery for resectable pancreatic cancer (Prep-02/JSAP05). Jpn J Clin Oncol 2019;49:190-4.

31. Versteijne E, Suker M, Groothuis K, et al. Preoperative chemoradiotherapy versus immediate surgery for resectable and borderline resectable pancreatic cancer: results of the Dutch Randomized Phase III PREOPANC trial. J Clin Oncol 2020;38: 1763-73.

32. Sohal D, Duong MT, Ahmad SA, et al. SWOG S1505: Results of perioperative chemotherapy (peri-op CTx) with mfolfirinox versus gemcitabine/nab-paclitaxel (Gem/nabP) for resectable pancreatic ductal adenocarcinoma (PDA). J Clin Oncol 2020;38(Suppl 15):4504.

33. Conroy T, Hammel P, Hebbar M, et al. FOLFIRINOX or gemcitabine as adjuvant therapy for pancreatic cancer. N Engl J Med 2018;379:2395-406.

34. Regine WF, Winter KA, Abrams R, et al. Fluorouracil-based chemoradiation with either gemcitabine or fluorouracil chemotherapy after resection of pancreatic adenocarcinoma: 5-year analysis of the U.S. Intergroup/RTOG 9704 phase III trial. Ann Surg Oncol 2011;18:1319-26.

35. Butturini G, Stocken DD, Wente MN, et al. Influence of resection margins and treatment on survival in patients with pancreatic cancer: meta-analysis of randomized controlled trials. Arch Surg 2008;143:75-83, discussion 83.

36. Merkow RP, Bilimoria KY, Tomlinson JS, et al. Postoperative complications reduce adjuvant chemotherapy use in resectable pancreatic cancer. Ann Surg 2014;260:372-7.

37. Tummers WS, Groen JV, Sibinga Mulder BG, et al. Impact of resection margin status on recurrence and survival in pancreatic cancer surgery. Br J Surg 2019; 106:1055-65.

38. Renouf DJ, Knox JJ, Kavan P, et al. The Canadian Cancer Trials Group PA.7 trial: Results of a randomized phase II study of gemcitabine (GEM) and nabpaclitaxel (Nab-P) vs GEM, nab-P, durvalumab (D) and tremelimumab $(T)$ as first line therapy in metastatic pancreatic ductal adenocarcinoma (mPDAC). Proceedings of the European Society of Medical Oncology (ESMO) Virtual Congress 2020; 2020 Sept. 19-21.

39. Lu DS, Reber HA, Krasny RM, et al. Local staging of pancreatic cancer: criteria for unresectability of major vessels as revealed by pancreatic-phase, thinsection helical CT. AJR Am J Roentgenol 1997;168:1439-43.

40. Varadhachary GR, Tamm EP, Abbruzzese JL, et al. Borderline resectable pancreatic cancer: definitions, management, and role of preoperative therapy. Ann Surg Oncol 2006;13:1035-46.

41. Kim EJ, Ben-Josef E, Herman JM, et al. A multi-institutional phase 2 study of neoadjuvant gemcitabine and oxaliplatin with radiation therapy in patients with pancreatic cancer. Cancer 2013;119:2692-700.

42. Katz MH, Shi Q, Ahmad SA, et al. Preoperative modified FOLFIRINOX treatment followed by capecitabine-based chemoradiation for borderline resectable pancreatic cancer: Alliance for Clinical Trials in Oncology Trial A021101. JAMA Surg 2016;151:e161137. 
43. Yoo C, Kang J, Kim KP, et al. Efficacy and safety of neoadjuvant FOLFIRINOX for borderline resectable pancreatic adenocarcinoma: improved efficacy compared with gemcitabine-based regimen. Oncotarget 2017;8:46337-47.

44. Fiore M, Ramella S, Valeri S, et al. Phase II study of induction chemotherapy followed by chemoradiotherapy in patients with borderline resectable and unresectable locally advanced pancreatic cancer. Sci Rep 2017;7:45845.

45. Janssen QP, Buettner S, Suker M, et al. Neoadjuvant FOLFIRINOX in patients with borderline resectable pancreatic cancer: a systematic review and patientlevel meta-analysis. J Natl Cancer Inst 2019;111:782-94.

46. Katz MH, Fleming JB, Bhosale $\mathrm{P}$, et al. Response of borderline resectable pancreatic cancer to neoadjuvant therapy is not reflected by radiographic indicators. Cancer 2012;118:5749-56.

47. Festa V, Andriulli A, Valvano MR, et al. Neoadjuvant chemo-radiotherapy for patients with borderline resectable pancreatic cancer: a meta-analytical evaluation of prospective studies. JOP 2013;14:618-25.

48. Katz MH, Crane CH, Varadhachary G. Management of borderline resectable pancreatic cancer. Semin Radiat Oncol 2014;24:105-12.

49. Murphy JE, Wo JY, Ryan DP, et al. Total neoadjuvant therapy with FOLFIRINOX followed by individualized chemoradiotherapy for borderline resectable pancreatic adenocarcinoma: a phase 2 clinical trial. JAMA Oncol 2018;4:963-9.

50. Hurt CN, Falk S, Crosby $\mathrm{T}$, et al. Long-term results and recurrence patterns from SCALOP: a phase II randomised trial of gemcitabine- or capecitabinebased chemoradiation for locally advanced pancreatic cancer. Br J Cancer 2017;116:1264-70.

51. Huguet F, Girard N, Guerche CS, et al. Chemoradiotherapy in the management of locally advanced pancreatic carcinoma: a qualitative systematic review. J Clin Oncol 2009;27:2269-77.

52. Suker M, Beumer BR, Sadot E, et al. FOLFIRINOX for locally advanced pancreatic cancer: a systematic review and patient-level meta-analysis. Lancet Oncol 2016;17:801-10.

53. Philip PA, Lacy J, Portales F, et al. Nab-paclitaxel plus gemcitabine in patients with locally advanced pancreatic cancer (LAPACT): a multicentre, open-label phase 2 study. Lancet Gastroenterol Hepatol 2020;5:285-94.

54. Willett CG, Czito BG, Bendell JC, et al. Locally advanced pancreatic cancer. J Clin Oncol 2005;23:4538-44.

55. lacobuzio-Donahue CA, Fu B, Yachida S, et al. DPC4 gene status of the primary carcinoma correlates with patterns of failure in patients with pancreatic cancer. J Clin Oncol 2009;27:1806-13.

56. Hoyer M, Roed H, Sengelov L, et al. Phase-II study on stereotactic radiotherapy of locally advanced pancreatic carcinoma. Radiother Oncol 2005;76:48-53.

57. Martin RC II, McFarland K, Ellis S, et al. Irreversible electroporation in locally advanced pancreatic cancer: potential improved overall survival. Ann Surg Oncol 2013;20(Suppl 3):S443-9.

58. Moris D, Machairas N, Tsilimigras DI, et al. Systematic review of surgical and percutaneous irreversible electroporation in the treatment of locally advanced pancreatic cancer. Ann Surg Oncol 2019;26:1657-68.

59. Ruarus AH, Vroomen L, Geboers B, et al. Percutaneous Irreversible Electroporation in Locally Advanced and Recurrent Pancreatic Cancer (PANFIRE-2): a multicenter, prospective, single-arm, phase II study. Radiology 2020;294:212-20.
60. Wang-Gillam A, Li CP, Bodoky G, et al. Nanoliposomal irinotecan with fluorouracil and folinic acid in metastatic pancreatic cancer after previous gemcitabine-based therapy (NAPOLI-1): a global, randomised, open-label, phase 3 trial. Lancet 2016;387:545-57.

61. O'Kane GM, Grunwald BT, Jang GH, et al. GATA6 expression distinguishes clas sical and basal-like subtypes in advanced pancreatic cancer. Clin Cancer Res 2020; 26:4901-10.

62. Chan-Seng-Yue M, Kim JC, Wilson GW, et al. Transcription phenotypes of pancreatic cancer are driven by genomic events during tumor evolution. Nat Genet 2020;52:231-40.

63. Connor AA, Denroche RE, Jang GH, et al. Integration of genomic and transcriptional features in pancreatic cancer reveals increased cell cycle progression in metastases. Cancer Cell 2019;35:267-282 e7.

64. Canon J, Rex K, Saiki AY, et al. The clinical KRAS(G12C) inhibitor AMG 510 drives anti-tumour immunity. Nature 2019;575:217-23.

65. Aung KL, Fischer SE, Denroche RE, et al. Genomics-driven precision medicine for advanced pancreatic cancer: early results from the COMPASS trial. Clin Cancer Res 2018;24:1344-54.

66. Aguirre AJ, Nowak JA, Camarda ND, et al. Real-time genomic characterization of advanced pancreatic cancer to enable precision medicine. Cancer Discov 2018;8:1096-111.

67. Drilon A, Laetsch TW, Kummar S, et al. Efficacy of larotrectinib in TRK fusionpositive cancers in adults and children. N Engl J Med 2018;378:731-9.

68. Jones MR, Williamson LM, Topham JT, et al. NRG1 gene fusions are recurrent, clinically actionable gene rearrangements in KRAS wild-type pancreatic ductal adenocarcinoma. Clin Cancer Res 2019;25:4674-81.

69. Heining $\mathrm{C}$, Horak $\mathrm{P}$, Uhrig $\mathrm{S}$, et al. NRG1 fusions in KRAS wild-type pancreatic cancer. Cancer Discov 2018;8:1087-95.

70. Singhi AD, Ali SM, Lacy J, et al. Identification of targetable ALK rearrangements in pancreatic ductal adenocarcinoma. J Natl Compr Canc Netw 2017;15:555-62.

71. Lowery MA, Jordan EJ, Basturk O, et al. Real-time genomic profiling of pancreatic ductal adenocarcinoma: potential actionability and correlation with clinical phenotype. Clin Cancer Res 2017;23:6094-100.

72. Pishvaian MJ, Blais EM, Brody JR, et al. Overall survival in patients with pancreatic cancer receiving matched therapies following molecular profiling: a retrospective analysis of the Know Your Tumor registry trial. Lancet Oncol 2020;21:508-18.

73. Hosein AN, Brekken RA, Maitra A. Pancreatic cancer stroma: an update on therapeutic targeting strategies. Nat Rev Gastroenterol Hepatol 2020;17:487-505.

74. Tempero MA, Van Cutsem E, Sigal D, et al.; HALO 109-301 Investigators. A randomized, double-blind, placebo-controlled, phase 3 study of pegvorhyaluronidase alfa (PEGPH20)+ nab-paclitaxel/gemcitabine (AG) in patients (pts) with previously untreated hyaluronan (HA)-high metastatic pancreatic ductal adenocarcinoma (mPDA). J Clin Oncol 2020;38(Suppl 4):638.

75. Lee $B$, Wong $A$, Kee $D$, et al. The use of ipilimumab in patients with rheumatoid arthritis and metastatic melanoma. Ann Oncol 2016;27:1174-7.

76. Le DT, Durham JN, Smith KN, et al. Mismatch repair deficiency predicts response of solid tumors to PD-1 blockade. Science 2017;357:409-13.

77. Marabelle A, Le DT, Ascierto PA, et al. Efficacy of pembrolizumab in patients with noncolorectal high microsatellite instability/mismatch repair-deficient cancer: results from the Phase II KEYNOTE-158 study. J Clin Oncol 2020;38:1-10.
Intérêts concurrents : Grainne O'Kane a reçu des honoraires de consultation et autres d'AstraZeneca, Roche et Eisai indépendamment des travaux soumis. Aucun autre intérêt concurrent déclaré.

Cet article a été commandé et a été révisé par les pairs.

Affiliations: Centre d'oncologie Princess Margaret (O'Kane, Gallinger), Réseau universitaire de santé de Toronto, Toronto, Ont.; Université de l'Alberta (Ladak), Edmonton, Alb.

Collaborateurs : Grainne O'Kane et Farah Ladak ont contribué à parts égales. Tous les auteurs ont substantiellement participé à la préparation du manuscrit, à la rédaction de l'ébauche et aux révisions; ils ont approuvé la version finale pour publication, se portent garants de tous les aspects du travail, et s'assurent que les questions relatives à l'exactitude ou à l'intégrité fassent l'objet d'une recherche appropriée.

Propriété intellectuelle du contenu : Il s'agit d'un article en libre accès distribué conformément aux modalités de la licence Creative Commons Attribution (CC BY-NC-ND 4.0), qui permet l'utilisation, la diffusion et la reproduction de tout médium à la condition que la publication originale soit adéquatement citée, que l'utilisation se fasse à des fins non commerciales (c.-à-d., recherche ou éducation) et qu'aucune modification ni adaptation n'y soit apportée. Voir : https://creativecommons.org/ licenses/by-nc-nd/4.0/deed.fr.

Correspondance : Grainne O’Kane, Grainne.O’Kane@uhn.ca 